\title{
Independent toxic effects of metallic compounds on Indian major carps
}

\author{
P. Senthilkumaar, S. Eswari, K. Velmurugan and S. Sarojini \\ School of Enzymology and Environmental Toxicology, Sir Theagaraya College, Chennai-600021, India \\ drsenthilkumaar@yahoo.co.in
}

\begin{abstract}
An attempt on individual toxicity of lead nitrate and barium chloride on the Indian major carps, catla, rohu and mrigal was carried out as they are the natural inhabitants of the perennial rivers network of India and neighbouring countries where pollutants are becoming a major stress factor for these commercially important edible fishes. For all the three major carps, the $96 \mathrm{hr} \mathrm{LC} 50$ and Sub Lethal Concentration (SLC) were in the order of Lead nitrate > Barium chloride. In the individual toxicity studies, the three carps exhibited a decreasing trend in total proteins and total lipids in all the test tissues but liver alone showed an increasing trend with reference to carbohydrate content.
\end{abstract}

Keywords: Catla, Rohu, Mrigal, Lead nitrate, Proteins, Carbohydrates and Lipids.

Introduction

Aquatic water bodies are frequently polluted with a multitude of potentially hazardous substances (Battaglin and Fairchild, 2002). Thus, protecting the integrity of our water resources is one of the most essential environmental issues of the 21st century. Recently, it has brought increasing concerns for potential adverse ecological health effects resulting from the production, use, and disposal of numerous chemicals that offer improvements in industry, agriculture, medical treatment, and even common household conveniences (Daughton \& Ternes, 1999). Heavy metals like lead are one of the major components of the industrial wastes, which along with other products from industrial operations are discharged into the aquatic environment and it is toxic to aquatic life (Korai et al., 2008). Contamination by heavy metal in aquatic system is a severe problem and fish are more repeatedly exposed to these pollutants and may be taken in through gills, skin and contaminated foods (Ribeiroa et al., 2005; Ling et al., 2011). As an inexpensive, soluble salt of barium, barium chloride finds wide applications in industry; barium chloride is mainly used in the purification of brine solution in caustic chlorine plants and also in the manufacture of heat treatment salts, in the manufacture of pigments and in the manufacture of other barium salts (ATSDR, 2007). Hence, aquatic organisms are often exposed to mixtures of toxicants because it is believed that regardless of where the pollution occurs, it will eventually end up in the aquatic environment (Frat et al., 2011). The effect of pollution on the inhabiting species was found to be more in fresh water than in marine water (Koyama, 1997). Hence, fingerlings of the Indian major carps, Catla catla (catla), Labeo rohita (rohu) and Cirrhina mrigala (mrigal) was selected to assess the independent toxic effects of industrial effluents, lead nitrate and barium chloride.

\section{Materials and methods}

The fingerlings of the Indian major carps namely, Catla catla, Labeo rohita and Cirrhina mrigala were collected from the Tamil Nadu Fish Seed Farm Poondi, Thiruvallur Dist. They were quarantined and acclimatized to laboratory condition for three days. During this period they were fed with groundnut oil cake and the water was also renewed alternatively after feeding. The environmental parameters

Research article

(CIndian Society for Education and Environment (iSee) like the temperature, $\mathrm{pH}$, salinity and dissolved oxygen of the water used were well within the acceptable range.

Healthy fishes without any observable pathological symptoms were chosen for the experiments. Fishes were divided into groups of ten each and exposed to the toxicants viz. Lead nitrate and Barium chloride independently. In the independent toxicity experiments, fingerlings of each species of Indian major carps were exposed to the test toxicants individually.

\section{Independent toxicity}

Initially the fingerlings of three species of Indian major carps were treated with lead nitrate and barium chloride individually to determine $96 \mathrm{hr} \mathrm{LC}_{50}$ values for the test toxicants. Based on the range finding bioassay, five different concentrations of investigative toxicants were prepared by dissolving in distilled water. Suitable controls without toxicant were maintained for all the experimental groups. Mortality was recorded for each day and tabulated to arrive at the cumulative per cent mortality at the end of $96 \mathrm{hr}$ for the different groups and $96 \mathrm{hr} \mathrm{LC}_{50}$ value was arrived using Finney (1971) probit analysis method.

One fourth of $\mathrm{LC}_{50}$ values obtained from the above experiments was taken as the Sub Lethal Concentration (SLC). Apparently normal and healthy fingerlings of catla, rohu and mrigal were exposed to these concentrations of the toxicant in groups for further experimental studies and sacrificed on $4^{\text {th }}, 7$ th, 14th, 21th and $28^{\text {th }}$ day of post exposure to study the biochemical parameters; total proteins, total carbohydrates and total lipids of fish muscle, liver, brain and kidney.

The proteins, carbohydrates and lipids, were assessed by the procedures of Carrol et al., (1956), Lowry et al., (1951) and Jayaraman (2003) respectively.

Data analysis was carried out using SPSS statistical package to arrive at mean, standard deviation and test of significance ( $P$ value).

Results and discussion

Determination of $96 \mathrm{hr} L C_{50}$ values for the test toxicants and fixing of sub lethal concentration (SLC) 
Vol. 5 No. 8 (August 2012) ISSN: 0974-6846

Catla catla: Fish was exposed to barium chloride in the range of $100-400 \mathrm{ppm}$. $50 \%$ mortality was noticed at 400 $\mathrm{ppm}$, which was taken as $96 \mathrm{hr} \mathrm{LC}_{50}$ for the toxicant.SLC was calculated as $100 \mathrm{ppm}$ being 1/4th of LC50 value (Table 1). Exposure to lead nitrate registered $50 \%$ mortality in $150 \mathrm{ppm}$ and SLC was calculated as $37.5 \mathrm{ppm}$ being 1/4th of $\mathrm{LC}_{50}$ value (Table 2 ).

Labeo rohita: Exposure to barium chloride in the range of $100-400 \mathrm{ppm}$ exhibited $50 \%$ mortality at $400 \mathrm{ppm}$, which was taken as $96 \mathrm{hr} \mathrm{LC}_{50}$ for the toxicant.SLC was calculated as $100 \mathrm{ppm}$ being 1/4th of LC $_{50}$ value (Table 3).

The fish was exposed to lead nitrate in the range of $150-400 \mathrm{ppm}$ to determine $96 \mathrm{hr}$ LC $_{50}$ concentration. $50 \%$ mortality was recorded in the experimental group subjected to $350 \mathrm{ppm}$ of the chemical. $87.5 \mathrm{ppm}$ being

Table 1. Determination of $96 \mathrm{hr} L C_{50}$ for Barium chloride

\begin{tabular}{|c|c|c|c|c|c|}
\hline Conc. & \multicolumn{4}{|c|}{ Percentage mortality } & Cumulative \\
\cline { 2 - 5 }$(\mathrm{ppm})$ & Day 1 & Day 2 & Day 3 & Day 4 & Mortality \\
\hline 100 & 0 & 0 & 0 & 0 & 0 \\
\hline 200 & 0 & 0 & 10 & 10 & 20 \\
\hline 300 & 0 & 0 & 10 & 20 & 30 \\
\hline 400 & 10 & 10 & 20 & 10 & 50 \\
\hline
\end{tabular}

Table 2. Determination of $96 \mathrm{hr} \angle C_{50}$ for Lead nitrate

\begin{tabular}{|c|c|c|c|c|c|}
\hline \multirow{2}{*}{$\begin{array}{l}\text { Conc. } \\
\text { (ppm) }\end{array}$} & \multicolumn{4}{|c|}{ Percentage mortality } & \multirow{2}{*}{$\begin{array}{l}\text { Cumulative } \\
\% \text { Mortality }\end{array}$} \\
\hline & Day 1 & Day 2 & Day 3 & Day 4 & \\
\hline 30 & 0 & 0 & 0 & 0 & 0 \\
\hline 60 & 0 & 0 & 10 & 10 & 20 \\
\hline 90 & 0 & 0 & 10 & 20 & 30 \\
\hline 120 & 0 & 10 & 20 & 10 & 40 \\
\hline 150 & 10 & 10 & 10 & 20 & 50 \\
\hline
\end{tabular}

Table 3. Determination of $96 \mathrm{hr} L C_{50}$ for Barium chloride

\begin{tabular}{|c|c|c|c|c|c|}
\hline $\begin{array}{c}\text { Conc. } \\
\text { (ppm) }\end{array}$ & \multicolumn{4}{|c|}{ Percentage mortality } & $\begin{array}{c}\text { Cumulative } \\
\text { \% Mortality }\end{array}$ \\
\hline 100 & 0 & 0 & 0 & 0 & 0 \\
\hline 200 & 0 & 0 & 10 & 10 & 20 \\
\hline 300 & 0 & 0 & 10 & 20 & 30 \\
\hline 400 & 10 & 10 & 20 & 10 & 50 \\
\hline
\end{tabular}

Table 4. Determination of $96 \mathrm{hr} L C_{50}$ for Lead nitrate

\begin{tabular}{|c|c|c|c|c|c|}
\hline \multirow{2}{*}{$\begin{array}{l}\text { Conc. } \\
\text { (ppm) }\end{array}$} & \multicolumn{4}{|c|}{ Percentage mortality } & \multirow{2}{*}{$\begin{array}{l}\text { Cumulative } \\
\% \text { Mortality }\end{array}$} \\
\hline & Day 1 & Day 2 & Day 3 & Day 4 & \\
\hline 150 & 0 & 0 & 0 & 0 & 0 \\
\hline 200 & 0 & 0 & 10 & 10 & 20 \\
\hline 250 & 0 & 0 & 10 & 20 & 30 \\
\hline 300 & 0 & 10 & 20 & 10 & 40 \\
\hline 350 & 10 & 10 & 10 & 20 & 50 \\
\hline
\end{tabular}

$1 / 4^{\text {th }}$ of $L C_{50}$ value was taken as SLC for lead nitrate toxicity studies (Table 4).

Cirrhina mrigala: 100-400 ppm range was designed for barium chloride noxiousness study. $400 \mathrm{ppm}$ concentration showed $50 \%$ death which was taken as $96 \mathrm{hr} \mathrm{LC}_{50}$ for the toxicant and SLC was calculated as 100 ppm being $1 / 4$ th of $\mathrm{LC}_{50}$ value (Table 5 ). For lead nitrate, $50 \%$ mortality was recorded at $300 \mathrm{ppm}$ concentration. The toxicant used was in the range of $150-300 \mathrm{ppm}$. The calculated SLC was 75 ppm (Table 6).
Table 5. Determination of $96 \mathrm{hr} L C_{50}$ for Barium chloride

\begin{tabular}{|c|c|c|c|c|c|c|}
\hline S. & Conc. & \multicolumn{4}{|c|}{ Percentage mortality } & Cumulative \\
No & $(\mathrm{ppm})$ & Day 1 & Day 2 & Day 3 & Day 4 & \\
\hline 1 & 100 & 0 & 0 & 0 & 0 & 0 \\
\hline 2 & 200 & 0 & 0 & 0 & 10 & 10 \\
\hline 3 & 300 & 0 & 0 & 0 & 20 & 20 \\
\hline 4 & 400 & 0 & 0 & 10 & 40 & 50 \\
\hline
\end{tabular}

Table 6. Determination of $96 \mathrm{hr} L C_{50}$ for Lead nitrate

\begin{tabular}{|c|c|c|c|c|c|c|}
\hline S. & Conc. & \multicolumn{4}{|c|}{ Percentage mortality } & Cumulative \\
\cline { 3 - 6 } No & $(\mathrm{ppm})$ & Day 1 & Day 2 & Day 3 & Day 4 & Mortality \\
\hline 1 & 150 & 0 & 0 & 0 & 0 & 0 \\
\hline 2 & 200 & 0 & 0 & 0 & 10 & 10 \\
\hline 3 & 250 & 0 & 0 & 20 & 10 & 30 \\
\hline 4 & 300 & 0 & 0 & 10 & 40 & 50 \\
\hline
\end{tabular}

Independent toxic effects on the nutritional content

Total proteins: The fingerlings of three species of Indian major carps exhibited a decreasing trend with reference to total protein content in the assessed tissues during the exposure periods of the tested toxicants (Tables 7-9).

Total carbohydrates

Barring the liver of the three species, the other tissues showed a declining trend in their total carbohydrate content during the experimental periods (Tables 10-12).

\section{Total lipids}

The dissected tissues of the fingerlings of the three species recorded a gradual decrease in their total lipid content during the exposure periods of the study toxicants (Tables 13-15).

Although scientists generally have a good knowledge of the toxicity of individual chemical contaminants, there is a great need to bridge the gap between our understanding of the toxic effects of exposure to individual xenobiotics and those effects from exposure to mixtures of such chemicals (Olmstead \& LeBlanc, 2005; Lister et al., 2011). To substantiate the nature of toxicity, assessment of biochemical parameters; proteins, carbohydrates and lipids are important as they indicate the vulnerability of organ systems to the pollutants (Fahmy, 2012). In the current research, these metabolic parameters have been utilised to detect the sub lethal effects of the investigative toxicants. Initially, the $\mathrm{LC}_{50}$ for two toxicants were determined independently. For all the three major carps, the $96 \mathrm{hr} \mathrm{LC}_{50}$ and Sub Lethal Concentration (SLC) were in the order of Lead nitrate > Barium chloride. Later in the individual toxicity studies, the three Indian major carps exhibited a decreasing trend in total proteins and total lipids in all the test tissues but liver alone showed an increasing trend with reference to carbohydrate content. The toxicity responses exhibited by the carps in this study are similar to those reported by other authors under various stress conditions (Palanivelu et al., 2005; Ufodike \& Onssiriuka, 2008;
Senthilkumaar et al. Indian J.Sci.Technol. 
Table 7 Catla catla: TOTAL PROTEINS ( $\mathrm{mg} / \mathrm{g}$ wet wt.)

\begin{tabular}{|c|c|c|c|c|c|c|c|}
\hline \multicolumn{8}{|c|}{ MUSCLE } \\
\hline TOXICANT & & CONTROL & $4^{\text {th }}$ DAY & $7^{\text {th }}$ DAY & $14^{\text {th }}$ DAY & $21^{\text {st }}$ DAY & $28^{\text {th }} \mathrm{DAY}$ \\
\hline \multirow[t]{3}{*}{$\mathrm{BaCl}_{2}$} & MEAN & 8.30 & 6.69 & 6.49 & 4.58 & 4.18 & 8.33 \\
\hline & S.D & 0.43 & 1.00 & 1.02 & 0.95 & 0.94 & 1.11 \\
\hline & $P$ & - & 0.001 & 0.02 & 0.001 & 0.001 & NS \\
\hline \multirow[t]{3}{*}{$\mathrm{PbNO}_{3}$} & MEAN & 8.30 & 6.22 & 5.88 & 5.12 & 4.58 & 7.14 \\
\hline & S.D & 0.43 & 1.02 & 1.32 & 1.96 & 0.72 & 1.02 \\
\hline & $\mathrm{P}$ & - & 0.001 & 0.001 & 0.001 & 0.001 & NS \\
\hline \multicolumn{8}{|c|}{ LIVER } \\
\hline \multirow[t]{3}{*}{$\mathrm{BaCl}_{2}$} & MEAN & 28.01 & 20.77 & 17.00 & 14.64 & 9.70 & 27.32 \\
\hline & S.D & 2.18 & 1.39 & 1.14 & 1.22 & 1.93 & 2.59 \\
\hline & $\mathrm{P}$ & - & 0.001 & 0.001 & 0.001 & 0.001 & NS \\
\hline \multirow[t]{3}{*}{$\mathrm{PbNO}_{3}$} & MEAN & 28.01 & 25.08 & 17.34 & 11.46 & 9.05 & 10.62 \\
\hline & S.D & 2.18 & 1.77 & 1.52 & 1.02 & 0.73 & 1.08 \\
\hline & $\mathrm{P}$ & - & 0.02 & 0.001 & 0.001 & 0.001 & 0.001 \\
\hline \multicolumn{8}{|c|}{ BRAIN } \\
\hline \multirow[t]{3}{*}{$\mathrm{BaCl}_{2}$} & MEAN & 7.11 & 5.18 & 4.17 & 2.71 & 2.38 & 5.64 \\
\hline & S.D & 1.19 & 0.86 & 0.96 & 0.76 & 1.13 & 1.30 \\
\hline & $P$ & - & NS & 0.001 & 0.001 & 0.001 & NS \\
\hline \multirow[t]{3}{*}{$\mathrm{PbNO}_{3}$} & MEAN & 7.11 & 6.93 & 5.77 & 4.86 & 2.84 & 4.35 \\
\hline & S.D & 1.19 & 0.97 & 1.31 & 1.04 & 0.93 & 0.67 \\
\hline & $\mathrm{P}$ & - & NS & NS & NS & 0.001 & 0.001 \\
\hline \multicolumn{8}{|c|}{ KIDNEY } \\
\hline \multirow[t]{3}{*}{$\mathrm{BaCl}_{2}$} & MEAN & 25.54 & 22.10 & 20.30 & 18.18 & 16.08 & 17.40 \\
\hline & S.D & 3.98 & 0.97 & 0.80 & 0.98 & 0.72 & 1.29 \\
\hline & $\mathrm{P}$ & - & 0.05 & 0.05 & 0.001 & 0.001 & 0.001 \\
\hline \multirow[t]{3}{*}{$\mathrm{PbNO}_{3}$} & MEAN & 25.54 & 23.62 & 21.14 & 16.05 & 12.10 & 13.81 \\
\hline & S.D & 3.98 & 1.56 & 1.23 & 1.47 & 1.24 & 1.06 \\
\hline & $\mathrm{P}$ & - & NS & NS & 0.001 & 0.001 & 0.001 \\
\hline
\end{tabular}

Table 8. Labeo rohita: TOTAL PROTEINS (mg/g wet wt.)

\begin{tabular}{|c|c|c|c|c|c|c|c|}
\hline \multicolumn{8}{|c|}{ MUSCLE } \\
\hline TOXICANT & & CONTROL & $4^{\text {th }}$ DAY & $7^{\text {th } D A Y}$ & $14^{\text {th }}$ DAY & $21^{\text {st }} \mathrm{DAY}$ & $28^{\text {th }} \mathrm{DAY}$ \\
\hline \multirow[t]{3}{*}{$\mathrm{BaCl}_{2}$} & MEAN & 44.09 & 31.64 & 30.13 & 26.70 & 23.41 & 40.15 \\
\hline & S.D & 1.28 & 3.83 & 1.51 & 1.66 & 1.56 & 1.11 \\
\hline & $\mathrm{P}$ & - & 0.001 & 0.001 & 0.001 & 0.001 & NS \\
\hline \multirow[t]{3}{*}{$\mathrm{PbNO}_{3}$} & MEAN & 44.09 & 38.21 & 20.44 & 19.14 & 17.69 & 41.35 \\
\hline & S.D & 1.28 & 1.29 & 1.26 & 1.68 & 0.97 & 1.08 \\
\hline & $\mathrm{P}$ & - & 0.001 & 0.001 & 0.001 & 0.001 & 0.001 \\
\hline \multicolumn{8}{|c|}{ LIVER } \\
\hline \multirow[t]{3}{*}{$\mathrm{BaCl}_{2}$} & MEAN & 20.27 & 18.30 & 17.10 & 16.28 & 11.13 & 18.85 \\
\hline & S.D & 1.43 & 1.29 & 1.45 & 1.09 & 0.94 & 1.83 \\
\hline & $\mathrm{P}$ & - & NS & 0.001 & 0.001 & 0.001 & 0.001 \\
\hline \multirow[t]{3}{*}{$\mathrm{PbNo}_{3}$} & MEAN & 20.27 & 15.48 & 13.29 & 9.78 & 6.01 & 18.76 \\
\hline & S.D & 1.43 & 1.90 & 1.32 & 1.38 & 1.37 & 1.48 \\
\hline & $\mathrm{P}$ & - & 0.001 & 0.001 & 0.001 & 0.001 & NS \\
\hline \multicolumn{8}{|c|}{ BRAIN } \\
\hline \multirow[t]{3}{*}{$\mathrm{BaCl}_{2}$} & MEAN & 9.91 & 7.22 & 5.41 & 3.07 & 2.13 & 6.08 \\
\hline & S.D & 0.94 & 0.90 & 1.26 & 0.45 & 0.21 & 1.72 \\
\hline & $\mathrm{P}$ & - & 0.001 & 0.001 & 0.001 & 0.001 & 0.001 \\
\hline \multirow[t]{3}{*}{$\mathrm{PbNO}_{3}$} & MEAN & 9.91 & 7.84 & 6.32 & 3.13 & 2.11 & 5.77 \\
\hline & S.D & 0.94 & 1.11 & 0.72 & 0.91 & 0.99 & 1.25 \\
\hline & $\mathrm{P}$ & - & 0.02 & 0.001 & 0.001 & 0.001 & 0.001 \\
\hline \multicolumn{8}{|c|}{ KIDNEY } \\
\hline \multirow[t]{3}{*}{$\mathrm{BaCl}_{2}$} & MEAN & 15.06 & 13.46 & 12.60 & 8.59 & 7.19 & 12.72 \\
\hline & S.D & 1.75 & 1.47 & 1.49 & 0.58 & 0.97 & 2.44 \\
\hline & $\mathrm{P}$ & - & NS & 0.001 & 0.001 & 0.001 & NS \\
\hline \multirow[t]{3}{*}{$\mathrm{PbNO}_{3}$} & MEAN & 15.06 & 13.64 & 12.04 & 9.86 & 4.85 & 12.12 \\
\hline & S.D & 1.75 & 0.80 & 0.98 & 0.79 & 1.17 & 0.59 \\
\hline & $\mathrm{P}$ & - & NS & 0.02 & 0.001 & 0.001 & 0.001 \\
\hline
\end{tabular}


Table 9. Cirrhina mrigala: TOTAL PROTEINS (mg/g wet wt.)

\begin{tabular}{|c|c|c|c|c|c|c|c|}
\hline \multicolumn{8}{|c|}{ MUSCLE } \\
\hline TOXICANT & & CONTROL & $4^{\text {th }} \mathrm{DAY}$ & $7^{\text {th }}$ DAY & $14^{\text {th }}$ DAY & $21^{\text {st }}$ DAY & $28^{\text {th }}$ DAY \\
\hline \multirow[t]{3}{*}{$\mathrm{BaCl}_{2}$} & MEAN & 8.43 & 6.12 & 3.89 & 2.75 & 1.17 & 7.70 \\
\hline & S.D & 1.33 & 1.09 & 1.14 & 1.23 & 0.70 & 1.16 \\
\hline & $\mathrm{P}$ & - & NS & 0.001 & 0.001 & 0.001 & NS \\
\hline \multirow[t]{3}{*}{$\mathrm{PbNO}_{3}$} & MEAN & 8.43 & 6.11 & 5.28 & 5.15 & 4.89 & 7.05 \\
\hline & S.D & 1.33 & 1.29 & 0.61 & 0.91 & 1.29 & 1.09 \\
\hline & $\mathrm{P}$ & - & 0.001 & 0.001 & 0.001 & 0.001 & NS \\
\hline \multicolumn{8}{|c|}{ LIVER } \\
\hline \multirow[t]{3}{*}{$\mathrm{BaCl}_{2}$} & MEAN & 4.08 & 3.25 & 2.87 & 2.58 & 1.32 & 3.79 \\
\hline & S.D & 1.00 & 1.05 & 0.98 & 1.19 & 0.66 & 1.34 \\
\hline & $\mathrm{P}$ & - & NS & NS & NS & 0.001 & NS \\
\hline \multirow[t]{3}{*}{$\mathrm{PbNO}_{3}$} & MEAN & 4.08 & 3.06 & 2.56 & 1.66 & 1.27 & 3.80 \\
\hline & S.D & 1.00 & 1.07 & 0.82 & 0.51 & 0.81 & 1.05 \\
\hline & $\mathrm{P}$ & - & NS & 0.05 & 0.001 & 0.001 & NS \\
\hline \multicolumn{8}{|c|}{ BRAIN } \\
\hline \multirow[t]{3}{*}{$\mathrm{BaCl}_{2}$} & MEAN & 6.01 & 5.81 & 5.15 & 3.98 & 2.58 & 5.16 \\
\hline & S.D & 0.95 & 0.98 & 1.01 & 1.27 & 1.19 & 1.41 \\
\hline & $\mathrm{P}$ & - & NS & NS & 0.001 & 0.001 & NS \\
\hline \multirow[t]{3}{*}{$\mathrm{PbNO}_{3}$} & MEAN & 6.01 & 5.72 & 5.35 & 4.81 & 4.37 & 5.86 \\
\hline & S.D & 0.95 & 1.32 & 1.28 & 1.10 & 0.96 & 1.11 \\
\hline & $\mathrm{P}$ & - & 0.05 & 0.05 & 0.001 & 0.001 & 0.001 \\
\hline \multicolumn{8}{|c|}{ KIDNEY } \\
\hline \multirow[t]{3}{*}{$\mathrm{BaCl}_{2}$} & MEAN & 11.66 & 10.87 & 9.15 & 7.70 & 6.93 & 11.21 \\
\hline & S.D & 1.07 & 2.20 & 1.18 & 1.16 & 1.14 & 0.83 \\
\hline & $\mathrm{P}$ & - & NS & NS & NS & 0.001 & NS \\
\hline \multirow[t]{3}{*}{$\mathrm{PbNO}_{3}$} & MEAN & 11.66 & 10.81 & 10.23 & 9.64 & 7.87 & 11.28 \\
\hline & S.D & 1.17 & 1.08 & 1.01 & 0.65 & 1.08 & 0.76 \\
\hline & $\mathrm{P}$ & - & 0.001 & 0.001 & 0.001 & 0.001 & NS \\
\hline
\end{tabular}

Table 10. Catla catla: TOTAL CARBOHYDRATES (mg/g wet wt.)

\begin{tabular}{|c|c|c|c|c|c|c|c|}
\hline \multicolumn{8}{|c|}{ MUSCLE } \\
\hline TOXICANT & & CONTROL & $4^{\text {th }}$ DAY & $7^{\text {th }}$ DAY & $14^{\text {th }}$ DAY & $21^{\text {st }}$ DAY & $28^{\text {th }} \mathrm{DAY}$ \\
\hline \multirow[t]{3}{*}{$\mathrm{BaCl}_{2}$} & MEAN & 0.97 & 0.81 & 0.72 & 0.60 & 0.58 & 0.73 \\
\hline & S.D & 0.11 & 0.07 & 0.07 & 0.08 & 0.04 & 0.08 \\
\hline & $\mathrm{P}$ & - & 0.001 & 0.001 & 0.001 & 0.001 & 0.001 \\
\hline \multirow[t]{3}{*}{$\mathrm{PbNO}_{3}$} & MEAN & 0.97 & 0.81 & 0.73 & 0.60 & 0.42 & 0.80 \\
\hline & S.D & 0.11 & 0.07 & 0.07 & 0.15 & 0.17 & 0.24 \\
\hline & $\mathrm{P}$ & - & 0.001 & 0.001 & 0.001 & 0.001 & NS \\
\hline \multicolumn{8}{|c|}{ LIVER } \\
\hline \multirow[t]{3}{*}{$\mathrm{BaCl}_{2}$} & MEAN & 26.25 & 26.75 & 27.01 & 27.95 & 28.35 & 25.32 \\
\hline & S.D & 1.15 & 2.18 & 1.13 & 1.06 & 1.17 & 1.46 \\
\hline & $\mathrm{P}$ & - & NS & 0.001 & 0.001 & 0.001 & NS \\
\hline \multirow[t]{3}{*}{$\mathrm{PbNO}_{3}$} & MEAN & 26.25 & 27.15 & 27.65 & 28.15 & 28.45 & 26.01 \\
\hline & S.D & 1.15 & 2.18 & 1.08 & 1.10 & 1.06 & 1.11 \\
\hline & $\mathrm{P}$ & - & NS & 0.001 & 0.001 & 0.001 & 0.001 \\
\hline \multicolumn{8}{|c|}{ BRAIN } \\
\hline \multirow[t]{3}{*}{$\mathrm{BaCl}_{2}$} & MEAN & 1.13 & 0.93 & 0.75 & 0.67 & 0.59 & 1.06 \\
\hline & S.D & 0.15 & 0.34 & 0.04 & 0.10 & 0.07 & 0.27 \\
\hline & $\mathrm{P}$ & - & NS & 0.001 & 0.001 & 0.001 & NS \\
\hline \multirow[t]{3}{*}{$\mathrm{PbNO}_{3}$} & MEAN & 1.13 & 0.98 & 0.63 & 0.56 & 0.23 & 1.06 \\
\hline & S.D & 0.15 & 0.28 & 0.11 & 0.09 & 0.09 & 0.31 \\
\hline & $\mathrm{P}$ & - & NS & 0.001 & 0.001 & 0.001 & NS \\
\hline \multicolumn{8}{|c|}{ KIDNEY } \\
\hline \multirow[t]{3}{*}{$\mathrm{BaCl}_{2}$} & MEAN & 2.86 & 1.66 & 1.53 & 1.12 & 0.88 & 1.66 \\
\hline & S.D & 0.41 & 1.09 & 0.40 & 0.62 & 0.28 & 1.09 \\
\hline & $\mathrm{P}$ & - & NS & 0.05 & 0.001 & 0.001 & NS \\
\hline \multirow[t]{3}{*}{$\mathrm{PbNO}_{3}$} & MEAN & 2.86 & 1.89 & 1.66 & 1.44 & 1.20 & 2.68 \\
\hline & S.D & 0.41 & 0.45 & 0.41 & 0.54 & 0.27 & 1.12 \\
\hline & $\mathrm{P}$ & - & 0.001 & 0.001 & 0.001 & 0.001 & NS \\
\hline
\end{tabular}


Table 11. Labeo rohita: TOTAL CARBOHYDRATES (mg/g wet wt.)

\begin{tabular}{|c|c|c|c|c|c|c|c|}
\hline \multicolumn{8}{|c|}{ MUSCLE } \\
\hline TOXICANT & & CONTROL & $4^{\text {th }}$ DAY & $7^{\text {th }}$ DAY & $14^{\text {th }} \mathrm{DAY}$ & $21^{\text {st }}$ DAY & $28^{\text {th }}$ DAY \\
\hline \multirow[t]{3}{*}{$\mathrm{BaCl}_{2}$} & MEAN & 3.87 & 2.03 & 0.90 & 0.86 & 0.76 & 0.84 \\
\hline & S.D & 0.30 & 0.85 & 0.10 & 0.06 & 0.05 & 0.18 \\
\hline & $\mathrm{P}$ & - & 0.001 & 0.001 & 0.001 & 0.001 & 0.001 \\
\hline \multirow[t]{3}{*}{$\mathrm{PbNO}_{3}$} & MEAN & 3.87 & 1.86 & 0.70 & 0.61 & 0.60 & 1.02 \\
\hline & S.D & 0.30 & 0.25 & 0.09 & 0.07 & 0.06 & 0.09 \\
\hline & $\mathrm{P}$ & - & 0.001 & 0.001 & 0.001 & 0.001 & 0.001 \\
\hline \multicolumn{8}{|c|}{ LIVER } \\
\hline \multirow[t]{3}{*}{$\mathrm{BaCl}_{2}$} & MEAN & 20.11 & 21.75 & 22.14 & 23.95 & 24.36 & 18.95 \\
\hline & S.D & 1.24 & 0.87 & 1.45 & 0.98 & 1.39 & 1.28 \\
\hline & $\mathrm{P}$ & - & 0.001 & 0.001 & 0.001 & 0.001 & 0.02 \\
\hline \multirow[t]{3}{*}{$\mathrm{PbNO}_{3}$} & MEAN & 20.11 & 21.95 & 22.35 & 22.93 & 23.15 & 19.75 \\
\hline & S.D & 1.24 & 1.41 & 1.46 & 1.23 & 0.85 & 1.02 \\
\hline & $P$ & - & 0.001 & 0.001 & 0.001 & 0.001 & 0.001 \\
\hline \multicolumn{8}{|c|}{ BRAIN } \\
\hline \multirow[t]{3}{*}{$\mathrm{BaCl}_{2}$} & MEAN & 1.83 & 0.95 & 0.91 & 0.77 & 0.46 & 0.76 \\
\hline & S.D & 0.44 & 0.10 & 0.10 & 0.07 & 0.19 & 0.05 \\
\hline & $\mathrm{P}$ & - & 0.001 & 0.001 & 0.001 & 0.001 & NS \\
\hline \multirow[t]{3}{*}{$\mathrm{PbNO}_{3}$} & MEAN & 1.83 & 1.39 & 0.71 & 0.66 & 0.61 & 1.46 \\
\hline & S.D & 0.44 & 0.10 & 0.06 & 0.08 & 0.10 & 0.10 \\
\hline & $\mathrm{P}$ & - & NS & 0.001 & 0.001 & 0.001 & NS \\
\hline \multicolumn{8}{|c|}{ KIDNEY } \\
\hline \multirow[t]{3}{*}{$\mathrm{BaCl}_{2}$} & MEAN & 5.75 & 3.94 & 2.19 & 1.62 & 1.37 & 3.14 \\
\hline & S.D & 1.07 & 0.33 & 0.11 & 0.06 & 0.13 & 1.10 \\
\hline & $P$ & - & 0.001 & 0.001 & 0.001 & 0.001 & NS \\
\hline \multirow[t]{3}{*}{$\mathrm{PbNO}_{3}$} & MEAN & 5.75 & 3.10 & 2.81 & 1.55 & 1.43 & 4.92 \\
\hline & S.D & 1.07 & 0.63 & 0.14 & 0.57 & 0.13 & 1.02 \\
\hline & $\mathrm{P}$ & - & 0.001 & 0.001 & 0.001 & 0.001 & NS \\
\hline
\end{tabular}

Table 12. Cirrhina mrigala: TOTAL CARBOHYDRATES (mg/g wet wt.)

\begin{tabular}{|c|c|c|c|c|c|c|c|}
\hline \multicolumn{8}{|c|}{ MUSCLE } \\
\hline TOXICANT & & CONTROL & $4^{\text {th }}$ DAY & $7^{\text {th } D A Y}$ & $14^{\text {th }}$ DAY & $21^{\text {st }}$ DAY & $28^{\text {th }} D A Y$ \\
\hline \multirow[t]{3}{*}{$\mathrm{BaCl}_{2}$} & MEAN & 2.49 & 1.84 & 1.74 & 1.35 & 1.15 & 2.36 \\
\hline & S.D & 0.72 & 0.83 & 0.96 & 0.56 & 0.42 & 1.40 \\
\hline & $\mathrm{P}$ & - & NS & NS & NS & 0.001 & NS \\
\hline \multirow[t]{3}{*}{$\mathrm{PbNO}_{3}$} & MEAN & 2.49 & 1.43 & 1.13 & 1.10 & 0.98 & 2.52 \\
\hline & S.D & 0.72 & 0.59 & 0.61 & 0.35 & 0.67 & 0.81 \\
\hline & $\mathrm{P}$ & - & NS & 0.001 & 0.001 & NS & NS \\
\hline \multicolumn{8}{|c|}{ LIVER } \\
\hline \multirow[t]{3}{*}{$\mathrm{BaCl}_{2}$} & MEAN & 23.50 & 24.51 & 25.10 & 25.87 & 26.35 & 21.09 \\
\hline & S.D & 0.81 & 0.97 & 2.90 & 0.98 & 1.02 & 1.15 \\
\hline & $\mathrm{P}$ & - & 0.001 & 0.001 & 0.001 & 0.001 & 0.001 \\
\hline \multirow[t]{3}{*}{$\mathrm{PbNO}_{3}$} & MEAN & 23.50 & 24.35 & 25.10 & 25.96 & 26.43 & 22.17 \\
\hline & S.D & 0.81 & 1.58 & 1.26 & 1.17 & 1.30 & 0.98 \\
\hline & $\mathrm{P}$ & - & 0.001 & 0.001 & 0.001 & 0.001 & 0.001 \\
\hline \multicolumn{8}{|c|}{ BRAIN } \\
\hline \multirow[t]{3}{*}{$\mathrm{BaCl}_{2}$} & MEAN & 3.54 & 2.60 & 1.81 & 1.05 & 1.32 & 3.14 \\
\hline & S.D & 0.87 & 1.13 & 1.01 & 0.70 & 0.72 & 1.10 \\
\hline & $\mathrm{P}$ & - & NS & NS & 0.001 & 0.001 & NS \\
\hline \multirow[t]{3}{*}{$\mathrm{PbNO}_{3}$} & MEAN & 3.54 & 2.35 & 2.11 & 1.76 & 1.68 & 3.21 \\
\hline & S.D & 0.87 & 0.91 & 1.43 & 1.08 & 1.08 & 0.87 \\
\hline & $P$ & - & NS & NS & 0.001 & NS & NS \\
\hline \multicolumn{8}{|c|}{ KIDNEY } \\
\hline \multirow[t]{3}{*}{$\mathrm{BaCl}_{2}$} & MEAN & 7.78 & 6.87 & 4.38 & 3.97 & 2.36 & 5.39 \\
\hline & S.D & 1.01 & 1.21 & 0.95 & 1.40 & 1.04 & 0.86 \\
\hline & $\mathrm{P}$ & - & NS & 0.001 & 0.001 & 0.001 & NS \\
\hline \multirow[t]{3}{*}{$\mathrm{PbNo}_{3}$} & MEAN & 7.78 & 4.07 & 3.33 & 2.87 & 2.65 & 5.69 \\
\hline & S.D & 1.01 & 1.01 & 1.41 & 0.86 & 1.15 & 1.01 \\
\hline & $\mathrm{P}$ & - & 0.001 & 0.001 & 0.001 & 0.001 & 0.001 \\
\hline
\end{tabular}

Research article Clndian Society for Education and Environment (iSee)
"Metallic toxicity in Indian fishes" http://www.indjst.org
Senthilkumaar et al. Indian J.Sci.Technol. 
Table 13. Catla catla: TOTAL LIPIDS (mg/g wet wt.)

\begin{tabular}{|c|c|c|c|c|c|c|c|}
\hline \multicolumn{8}{|c|}{ MUSCLE } \\
\hline TOXICANT & & CONTROL & $4^{\text {th }}$ DAY & $7^{\text {th }}$ DAY & $14^{\text {th }}$ DAY & $21^{\text {st }}$ DAY & $28^{\text {th }} D A Y$ \\
\hline \multirow[t]{3}{*}{$\mathrm{BaCl}_{2}$} & MEAN & 8.43 & 5.14 & 4.05 & 3.37 & 2.72 & 6.99 \\
\hline & S.D & 0.77 & 0.79 & 0.79 & 0.27 & 0.67 & 1.18 \\
\hline & $\mathrm{P}$ & - & 0.001 & 0.001 & 0.001 & 0.001 & NS \\
\hline \multirow[t]{3}{*}{$\mathrm{PbNO}_{3}$} & MEAN & 8.43 & 6.73 & 5.10 & 4.57 & 3.62 & 4.81 \\
\hline & S.D & 0.77 & 1.11 & 0.84 & 0.76 & 0.81 & 0.96 \\
\hline & $\mathrm{P}$ & - & NS & 0.001 & 0.001 & 0.001 & 0.001 \\
\hline \multicolumn{8}{|c|}{ LIVER } \\
\hline \multirow[t]{3}{*}{$\mathrm{BaCl}_{2}$} & MEAN & 8.38 & 6.79 & 6.00 & 3.40 & 2.48 & 7.10 \\
\hline & S.D & 1.14 & 1.02 & 1.00 & 0.71 & 0.72 & 1.04 \\
\hline & $\mathrm{P}$ & - & NS & 0.001 & 0.001 & 0.001 & NS \\
\hline \multirow[t]{3}{*}{$\mathrm{PbNO}_{3}$} & MEAN & 8.38 & 7.66 & 6.67 & 5.45 & 4.95 & 7.85 \\
\hline & S.D & 1.14 & 0.54 & 1.16 & 0.61 & 1.15 & 1.08 \\
\hline & $\mathrm{P}$ & - & NS & 0.05 & 0.001 & 0.001 & NS \\
\hline \multicolumn{8}{|c|}{ BRAIN } \\
\hline \multirow[t]{3}{*}{$\mathrm{BaCl}_{2}$} & MEAN & 7.55 & 5.14 & 4.58 & 4.36 & 3.41 & 6.67 \\
\hline & S.D & 1.47 & 0.79 & 0.95 & 1.27 & 1.01 & 1.16 \\
\hline & $\mathrm{P}$ & - & 0.001 & 0.02 & 0.05 & 0.001 & NS \\
\hline \multirow[t]{3}{*}{$\mathrm{PbNO}_{3}$} & MEAN & 7.55 & 5.41 & 4.77 & 3.65 & 4.36 & 7.28 \\
\hline & S.D & 1.47 & 0.76 & 0.89 & 0.40 & 1.60 & 0.84 \\
\hline & $\mathrm{P}$ & - & NS & 0.001 & 0.001 & 0.001 & NS \\
\hline \multicolumn{8}{|c|}{ KIDNEY } \\
\hline \multirow[t]{3}{*}{$\mathrm{BaCl}_{2}$} & MEAN & 4.76 & 3.92 & 2.48 & 1.32 & 0.99 & 1.55 \\
\hline & S.D & 0.58 & 0.95 & 0.60 & 0.66 & 0.28 & 0.56 \\
\hline & $\mathrm{P}$ & - & NS & 0.001 & 0.001 & 0.001 & 0.001 \\
\hline \multirow[t]{3}{*}{$\mathrm{PbNo}_{3}$} & MEAN & 4.76 & 3.87 & 3.33 & 2.54 & 1.87 & 4.17 \\
\hline & S.D & 0.58 & 1.00 & 0.92 & 0.82 & 0.72 & 0.94 \\
\hline & $\mathrm{P}$ & - & NS & 0.001 & 0.001 & 0.001 & NS \\
\hline
\end{tabular}

Table 14. Labeo rohita: TOTAL LIPIDS (mg/g wet wt.)

\begin{tabular}{|c|c|c|c|c|c|c|c|}
\hline \multicolumn{8}{|c|}{ MUSCLE } \\
\hline TOXICANT & & CONTROL & $4^{\text {th }}$ DAY & $7^{\text {th }}$ DAY & $14^{\text {th }} \mathrm{DAY}$ & $21^{\text {st }}$ DAY & $28^{\text {th }}$ DAY \\
\hline \multirow[t]{3}{*}{$\mathrm{BaCl}_{2}$} & MEAN & 3.66 & 3.07 & 2.79 & 2.47 & 1.81 & 3.40 \\
\hline & S.D & 0.41 & 0.45 & 0.35 & 0.73 & 0.58 & 1.04 \\
\hline & $\mathrm{P}$ & - & NS & 0.001 & NS & 0.05 & 0.001 \\
\hline \multirow[t]{3}{*}{$\mathrm{PbNO}_{3}$} & MEAN & 3.66 & 2.78 & 1.81 & 0.98 & 0.61 & 2.61 \\
\hline & S.D & 0.41 & 0.55 & 0.58 & 0.24 & 0.10 & 1.00 \\
\hline & $\mathrm{P}$ & - & NS & 0.001 & 0.001 & 0.001 & NS \\
\hline \multicolumn{8}{|c|}{ LIVER } \\
\hline \multirow[t]{3}{*}{$\mathrm{BaCl}_{2}$} & MEAN & 14.60 & 12.81 & 11.93 & 10.13 & 10.10 & 14.33 \\
\hline & S.D & 1.34 & 1.37 & 1.23 & 1.24 & 1.33 & 1.22 \\
\hline & $\mathrm{P}$ & - & 0.001 & 0.001 & 0.001 & 0.001 & NS \\
\hline \multirow[t]{3}{*}{$\mathrm{PbNO}_{3}$} & MEAN & 14.60 & 12.84 & 8.55 & 7.57 & 4.12 & 13.37 \\
\hline & S.D & 1.34 & 1.20 & 0.56 & 0.90 & 0.77 & 1.60 \\
\hline & $\mathrm{P}$ & - & NS & 0.001 & 0.001 & 0.001 & NS \\
\hline \multicolumn{8}{|c|}{ BRAIN } \\
\hline \multirow[t]{3}{*}{$\mathrm{BaCl}_{2}$} & MEAN & 19.00 & 18.90 & 15.10 & 13.01 & 11.12 & 18.22 \\
\hline & S.D & 1.15 & 1.18 & 1.04 & 0.99 & 1.14 & 1.09 \\
\hline & $\mathrm{P}$ & - & 0.001 & 0.001 & 0.001 & 0.001 & 0.001 \\
\hline \multirow[t]{3}{*}{$\mathrm{PbNO}_{3}$} & MEAN & 19.00 & 16.70 & 14.34 & 13.15 & 11.99 & 15.21 \\
\hline & S.D & 1.15 & 1.24 & 0.86 & 0.89 & 1.60 & 0.86 \\
\hline & $\mathrm{P}$ & - & NS & 0.001 & 0.001 & 0.001 & 0.001 \\
\hline \multicolumn{8}{|c|}{ KIDNEY } \\
\hline \multirow[t]{3}{*}{$\mathrm{BaCl}_{2}$} & MEAN & 10.68 & 7.15 & 5.41 & 4.37 & 3.95 & 9.38 \\
\hline & S.D & 1.09 & 1.45 & 1.26 & 0.87 & 0.32 & 1.04 \\
\hline & $\mathrm{P}$ & - & 0.001 & 0.001 & 0.001 & 0.001 & 0.001 \\
\hline \multirow[t]{3}{*}{$\mathrm{PbNO}_{3}$} & MEAN & 10.68 & 8.02 & 6.82 & 3.90 & 2.48 & 7.22 \\
\hline & S.D & 1.09 & 0.69 & 1.12 & 0.85 & 0.72 & 0.90 \\
\hline & $\mathrm{P}$ & - & 0.001 & 0.001 & 0.001 & 0.001 & 0.001 \\
\hline
\end{tabular}

Research article CIndian Society for Education and Environment (iSee)
"Metallic toxicity in Indian fishes" http://www.indjst.org
Senthilkumaar et al. Indian J.Sci.Technol. 
Vol. 5 No. 8 (August 2012) ISSN: 0974-6846

Table 15. Cirrhina mrigala: TOTAL LIPIDS (mg/g wet wt.)

\begin{tabular}{|c|c|c|c|c|c|c|c|}
\hline \multicolumn{8}{|c|}{ MUSCLE } \\
\hline TOXICANT & & CONTROL & $4^{\text {th }}$ DAY & $7^{\text {th }}$ DAY & $14^{\text {th }}$ DAY & $21^{\text {st }}$ DAY & $28^{\text {th }}$ DAY \\
\hline \multirow[t]{3}{*}{$\mathrm{BaCl}_{2}$} & MEAN & 3.82 & 3.00 & 2.76 & 1.26 & 1.05 & 3.57 \\
\hline & S.D & 0.94 & 1.11 & 1.62 & 0.37 & 0.22 & 0.51 \\
\hline & $\mathrm{P}$ & - & NS & NS & 0.001 & 0.001 & NS \\
\hline \multirow[t]{3}{*}{$\mathrm{PbNO}_{3}$} & MEAN & 3.82 & 2.36 & 2.64 & 1.59 & 1.14 & 3.33 \\
\hline & S.D & 0.94 & 0.84 & 1.10 & 0.34 & 0.49 & 0.68 \\
\hline & $\mathrm{P}$ & - & NS & NS & 0.001 & 0.001 & NS \\
\hline \multicolumn{8}{|c|}{ LIVER } \\
\hline \multirow[t]{3}{*}{$\mathrm{BaCl}_{2}$} & MEAN & 10.38 & 8.57 & 8.03 & 7.13 & 6.39 & 9.60 \\
\hline & S.D & 1.14 & 0.73 & 1.10 & 1.04 & 1.04 & 0.99 \\
\hline & $\mathrm{P}$ & - & 0.05 & 0.001 & 0.001 & 0.001 & NS \\
\hline \multirow[t]{3}{*}{$\mathrm{PbNO}_{3}$} & MEAN & 10.38 & 8.62 & 7.68 & 5.14 & 4.92 & 9.21 \\
\hline & S.D & 1.14 & 0.70 & 1.07 & 0.79 & 0.86 & 1.41 \\
\hline & $\mathrm{P}$ & - & 0.001 & 0.001 & 0.001 & 0.001 & NS \\
\hline \multicolumn{8}{|c|}{ BRAIN } \\
\hline \multirow[t]{3}{*}{$\mathrm{BaCl}_{2}$} & MEAN & 15.42 & 14.35 & 13.19 & 11.53 & 10.13 & 14.58 \\
\hline & S.D & 1.15 & 0.64 & 1.15 & 1.26 & 0.91 & 1.17 \\
\hline & $\mathrm{P}$ & - & NS & NS & 0.001 & 0.001 & NS \\
\hline \multirow[t]{3}{*}{$\mathrm{PbNO}_{3}$} & MEAN & 15.42 & 13.83 & 12.26 & 11.53 & 10.56 & 14.96 \\
\hline & S.D & 1.15 & 1.10 & 1.45 & 1.26 & 1.16 & 1.02 \\
\hline & $\mathrm{P}$ & - & NS & NS & 0.001 & 0.001 & NS \\
\hline \multicolumn{8}{|c|}{ KIDNEY } \\
\hline \multirow[t]{3}{*}{$\mathrm{BaCl}_{2}$} & MEAN & 34.26 & 18.91 & 16.31 & 13.02 & 11.06 & 20.15 \\
\hline & S.D & 1.33 & 1.48 & 1.24 & 1.08 & 1.07 & 1.73 \\
\hline & $\mathrm{P}$ & - & 0.001 & 0.001 & 0.001 & 0.001 & 0.001 \\
\hline \multirow[t]{3}{*}{$\mathrm{PbNO}_{3}$} & MEAN & 34.26 & 23.82 & 21.01 & 19.40 & 10.80 & 26.12 \\
\hline & S.D & 1.33 & 1.08 & 1.14 & 0.90 & 1.01 & 1.32 \\
\hline & $\mathrm{P}$ & - & 0.001 & 0.001 & 0.001 & 0.001 & 0.001 \\
\hline
\end{tabular}

Capkin et al., 2010). The reduced protein levels in pollutant stressed tissues strongly suggest the toxicant induced proteolysis to meet the increased energy demand. (Saravanan et al., 2000; Kumar et al., 2005). This non carbohydrate source of energy is achieved with the escalation of aspartate amino transferase (AST) and alanine amino transferase (ALT) in the stressed tissues of carps (Tilak et al., 2005; Saravanan, 2010). Thus proteins can be expected to be involved in the compensatory mechanism of stressed organisms (Fahmy, 2012). The lipid content decreased in all the test tissues during the experimental period for toxicants studied justify the utilization of energy storehouse to meet the necessity of more energy for detoxification process and also to balance the hindrance of normal metabolism (Remia, et al., 2008). The detoxifying role in combating xenobiotics of the liver was evident in the present experiment as it alone depicted a slightly elevated level of total carbohydrates during the post exposure of individual toxicants.

\section{Conclusion}

In a contaminated aquatic environment, addition of toxicants and its exposure to non-target organisms like fishes is becoming a continuous process. This constant environmental stress will have a detrimental effect on freshwater fishes and it is of grave concern when edible ones are affected. In the present study, this was evident in the depletion of nutritional content of fingerlings of three important Indian major carps when exposed to individual xenobiotics and the damage would be much more in combination effect (successive) and additive outcome would be obvious when the fishes were in continuous stress. Pesticide and heavy metal are known to accumulate even at the fingerlings stage and usually gets magnified in the adults. If this situation prevails, the consequence of the toxicants may be the production of less nutritive fish foods. Unless scientific monitoring of water bodies and the quality of aqua wealth, particularly the edible ones is undertaken, fish food health hazards are in the waiting for the present and future human population. The present finding is an alarming signal to the Indian community, which is already fronting many issues of nutrition concern. Finally, the alterations in the biochemical parameters as observed in the current investigation may be used as one of the biomarker against anthropogenic stress.

References

1. ATSDR (2007) Toxicological profile for barium and barium compounds. U.S. Department of Health and Human Services, Public Health Service, Agency for Toxic Substances and Disease Registry, Atlanta, GA.

2. Battaglin WA and Fairchild JF (2002) Potential toxicity of pesticides measured in mid-western streams to aquatic organisms. Water Sci. Technol. 45, 95-103.

3. Capkin E, Kayis S, Boran H and Altinok I (2010) Acute toxicity of some agriculture fertilizers to rain 
bow trout, Oncorhynchus mykiss. Turkish J. Fisheries \& Aquatic Sci. 10, 19-25.

4. Carrol WV, Longley RW and Roe JH (1956) The determination of glycogen in the liver and muscle by the use of anthrone reagent. J. Biol. Chem. 220, 583593.

5. Daughton CG and Ternes TA (1999) Pharmaceuticals and personal care products in the environment: Agents of subtle change? Environ. Health Perspect. 107, 907-938.

6. Fahmy H (2012) Malathion toxicity: Effect on some metabolic activities in Oreochromis niloticus, the Tilapia Fish. Gehan Intl. J. Biosci., Biochem. \& Bioinformatics. 2(1), 52-55.

7. Fiat $O$, Cogon HY, Yüzereroğlu TA, Gök G , Fırat $O$, Kargin $F$ and Kötemen $Y$ (2011) A comparative study on the effects of a pesticide (cypermethrin) and two metals (copper, lead) to serum biochemistry of Nile tilapia, Oreochromis niloticus. Fish Physiol. Biochem. 37(3), 657-666.

8. Finney DJ (1971) Probity analysis. Cambridge Univ. Press. 3rd Edition. pp: 333.

9. Jayaraman J (2003) Laboratory manual in biochemistry. New Delhi, New Age International Publ., Edition 1, pp:180.

10. Korai AL, Sahato GA, Kazi TG and Lashari KH (2008) Lead concentrations in fresh water, muscle, gill and liver of Catla Catla (Hamilton) from Keenjhar Lake. Pak. J. Anal. Environ. Chem. 9(1), 11-19.

11. Koyama J (1997) Difference of heavy metal toxicities for freshwater fish and marine fish. Bull. Nat. Rest. Ins. Fish. Sci. 10, 123-133.

12. Kumar P, Gupta YG, Tiwari V, Tiwari $S$ and Ajay Singh (2005) Haematological and biochemical abnormalities in Cirrhinus mrigala (Ham.) induced by lead. J. Ecophysiol. Occupl. Hlth. 5, 213-216.

13. Ling XP, Zhang YH, Lu YH and Huang HQ (2011) Superoxide dismutase, catalase and acetyl cholinesterase: biomarkers for the joint effects of cadmium, zinc and methyl parathion contamination in water. Environ. Technol. 32(13), 1463-1470.

14. Lister LJ, Svendsen C, Wright J, Hooper HL and Spurgeon DJ (2011) Modelling the joint effects of a metal and a pesticide on reproduction and toxicokinetics in Lumbricid earthworms. Environ In. 37(4), 663-670.

15. Lowry $\mathrm{OH}$, Rosebrough NJ, Farr AL and Randall RJ (1951) Protein measurement with folin phenol reagent. J. Bio. Chem. 193, 265-275.

16. Olmstead AW and LeBlanc GA (2005) Toxicity assessment of environmentally relevant pollutant mixtures using a heuristic model. Integr. Environ. Assess. Manag. 2, 114-122.

17. Palanivelu V, Vijayavel K, Ezhilarasibalasubramanian $S$ and Balasubramanian MP (2005) Impact of fertilizer (urea) on oxygen consumption and feeding the fresh water fish, Oreochromis mossambicus. Environ. Toxicol. \& Pharmacol. 19, 351-355.

18. Remia KM, Logaswamy S, Logankuma $\mathrm{K}$ and Rajmohan D (2008) Effect of an insecticide (Monocrotophos) on some biochemical constituents of the fish Tilipia Mossambica. Poll. Res. 27(3), 523526.

19. Ribeiroa CAO, Vollaireb $Y$, Sanchez-Chardic $A$ and Rocheb H (2005) Bioaccumulation and the effects of organochlorine pesticides, PAH and heavy metals in the eel (Anguilla anguilla) at the Camargue Nature Reserve, France. Aquatic Toxicol. 74 (1), 53-69.

20. Saravanan TS, Aneez Mohamed M and Harikrishnan R (2000) Studies on the chronic effects of endosulfan on blood and liver of Orechromis mossambicus. J. Ecol. Res. Biocon. 1, 24 -27.

21. Saravanan TS, Rajesh $P$ and Sundaramoorthy $M$ (2010) Studies on effects of chronic exposure of endosulfan to Labeo rohita. J. Environ. Biol. 31(5), 755-758.

22. Tilak KS, Veeraiah K and Koleswara Rao D (2005) Biochemical changes induced by chlorpyrifos, an organophosphate compound in sublethal concentrations to the freshwater fish Catla catla, Labeo rohita and Cirrhinus mrigala. J. Environ. Biol. 26, 341-347.

23. Ufodike EBC and Onusiriuka BC (2008) Acute toxicity of inorganic fertilizers to African catfish, Clarius gariepinus (Teugals). Aquaculture Res. 21, 181-186.
"Metallic toxicity in Indian fishes" http://www.indjst.org
Senthilkumaar et al. Indian J.Sci.Technol. 